\title{
Diferentes índices radiográficos versus Índice Acetabular en la decisión de colocación de Arnés de Pavlik en pacientes con diagnóstico de Displasia de Cadera
}

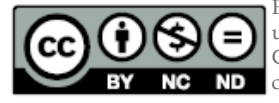

Este artículo está bajo una licencia de Creative Commons de tipo Reconoimiento - No comercial OPEN ACCESS Sin obras derivadas 4.0 International

1 Posgrado Traumatología y Ortopedia - Pontificia Universidad Católica del Ecuador (PUCE).

2 Médico tratante Traumatologia y Ortopedia - Hospital Vozandes Quito

ORCID ID:

Diego Silva Tirado:

https:/ / orcid.org/0000-0003-4223-0827

Francisco Vallejo Cifuentes:

https:// orcid.org/0000-0001-8448-9867

Received: 07 - Oct - 2019

Accepted: 20 - Dec - 2019

Publish: 27 - Dec - 2019

Corresponding author: Diego Silva Tirado

E-mail: diego.sebastian.silva.t@gmail.com

Authorship: Diego Silva and Francisco Vallejo conceptualized and designed the data, analyzed and interpreted the data, drafted the article, and critically revised the article.

Financial disclosure: The authors have no financial relationships relevant to this article to disclose.

STROBE 2008 Check List statement: The authors have real the STROBE 2008 Check List and the manuscript was prepared and revised according to the Strobe 2009 Checklist.

\section{Resumen}

Existen diferentes índices y clasificaciones radiográficas para el diagnóstico de Displasia del Desarrollo de la Cadera, este estudio comparó la utilidad de diferentes parámetros radiográficos en pacientes con índices acetabulares elevados a fin de determinar si se beneficiarán o no de un tratamiento ortopédico.

Pacientes y Métodos: Estudio de cohorte retrospectivo. Pacientes de 3 a 7 meses, atendidos en la consulta externa de Traumatología y Ortopedia pediátrica del Hospital Vozandes Quito en el período enero a diciembre de 2017 fueron incluidos en el estudio.

Signos de inestabilidad de la cadera al examen físico, mediciones radiográficas: índice acetabular, porcentaje de migración de cabeza femoral, índice de Smith, clasificación de Tonnis por cuadrantes, formación de la ceja acetabular radiográfica se obtuvieron del prontuario médico de cada paciente.

Resultados: Se evaluaron 104 pacientes, la mediana de la edad al momento del diagnóstico fue 4,63 meses, el $52 \%$ eran del sexo masculino y la media del índice acetabular fue de $37,9^{\circ}$.

Los pacientes fueron subdivididos en 2 grupos de acuerdo con el tratamiento ortopédico instaurado: con arnés de Pavlik $(38,5 \%)$ y sin arnés (61,5\%).

Al comparar pacientes sin y con arnés obtuvimos un OR de 1,07 y un valor $\mathrm{p}=0.69$

Pacientes con una escala de Tonnis grado I tuvieron $96 \%$ de probabilidad de desarrollar una cadera normal en relación con el grado II de Tonnis (60\%), con un OR de 21,3 y $p=0,69$.

Conclusión: Existe un grupo de pacientes con un índice acetabular elevado y caderas estables al examen físico que no se beneficiarían de un tratamiento ortopédico. Dentro de todos los parámetros evaluados observamos que la ceja acetabular radiográfica normal y la escala de Tonnis grado I fueron parámetros predictores de una buena respuesta a los 2 meses.

El índice de Smith y el porcentaje de Reimers no tiene valor pronóstico en pacientes con sospecha de displasia de cadera.

Palabras clave: Displasia del desarrollo de la cadera, equipos ortopédicos: Arnés de Pavlik, interpretación de imágenes: índice Acetabular, índice de Smith, porcentaje de Reimers, escala de Tonnis, ceja acetabular.
Citation: Silva D, Vallejo F. Diferentes índices radiográficos versus Índice Acetabular en la decisión de colocación de Arnés de Pavlik en pacientes con diagnóstico de Displasia de Cadera. Rev Med Vozandes. 2019; 30 (2): 35 - 41 
Keywords: Developmental Dysplasia of the Hip, Orthopedic Equipment: Pavlik harness, Image interpretations: acetabular index, Smith index, Reimers percentage, Tonnis scale, acetabular sourcil.

\section{Abstract}

\section{Different $X$-ray indices versus Acetabular Index on Pavlik Harness Placement Decision in Patients Diagnosed with Hip Dysplasia}

There are different $x$-ray indices and classifications for the diagnosis of Hip Developmental Dysplasia, this study compared the usefulness of different $x$-ray parameters in patients with high acetabular indices to determine whether or not would benefit from orthopedic treatment.

Patients and Methods: Retrospective cohort study. Patients aged 3 to 7 months, attended in the external consultation of Traumatology and Pediatric Orthopedics of the Hospital Vozandes Quito in the period January to December 2017 were included in the study.

Signs of hip instability to physical examination, radiographic measurements: acetabular index, percentage of femoral head migration, Smith index, Tonnis classification by quadrants, formation of the radiographic acetabular eyebrow were obtained from the medical record of each patient.

Results: 104 patients were evaluated, the median age at the time of diagnosis was 4.63 months, $52 \%$ were male and the mean acetabular index was 37.9 degrees.

Patients were subdivided into 2 groups according to the orthopedic treatment instituted: with Pavlik harness $(38.5 \%)$ and without a harness (61.5\%).

When comparing patients without and with harness we got an OR of 1.07 and a value of $\mathrm{p}-0.69$.

Patients with a Grade I Tonnis scale had a $96 \%$ chance of developing a normal hip relative to Tonnis Grade II (60\%), with an OR of 21.3 and p-0.69.

Conclusions: There is a group of patients with a high acetabular index and hips stable to the physical examination who would not benefit from orthopedic treatment, within all the parameters evaluated we observe that the normal radiographic acetabular eyebrow and the Tonnis scale grade I were predictor parameters of a good response at 2 months.

Smith's index and reimers percentage have no prognostic value in patients with suspected hip dysplasia. 


\section{Introducción}

La displasia del desarrollo de la cadera (DDC), comprende un espectro de anormalidades en la articulación coxofemoral: alteraciones congénitas, hereditarias y/o degenerativas.

Estás alteraciones producen cambios en la forma de la cabeza del fémur, del acetábulo o de ambos, provocando desde problemas del desarrollo e inestabilidad de la articulación, hasta graves alteraciones de la marcha, dolor y cojera a largo plazo; además de un desgaste excesivo de la articulación e invalidez. (1)

De forma general afecta de 1 a 4 por cada 1000 nacidos vivos, aunque su distribución varía entre las diferentes áreas geográficas, en México por ejemplo afecta de 2 a 6 infantes por cada mil nacidos vivos (2), en el Ecuador, estudio previo (3), realizado en un hospital de referencia reportó una prevalencia de DDC del 15,3\% en pacientes con edades comprendidas entre 3 y 6 meses atendidos en la consulta externa de Traumatología y Ortopedia pediátrica. ${ }^{(3)}$

La DDC es la principal causa de remplazo total de cadera en pacientes adultos jóvenes y representa del $21 \%$ al $29 \%$ de todos los casos intervenidos quirúrgicamente. ${ }^{(4)}$

El índice acetabular es el mejor parámetro para predecir displasia acetabular residual, y además sirve para determinar la evolución de los pacientes con displasia. ${ }^{(4,5)}$

Existe amplia controversia en cuanto al punto de corte del índice Acetabular para catalogar como una inclinación acetabular normal o patológica, resultando en un posible uso innecesario de dispositivos ortopédicos en pacientes con valores límite o ligeramente elevados.

El Arnés de Pavlik junto a otros dispositivos ortopédicos como el splint de Tubingen son los tratamientos más usados y recomendados en pacientes menores de 6 meses con una tasa de éxito de hasta el $80 \%{ }^{(6,7)}$, se recomienda la utilización de este dispositivo en edades tempranas incluso en pacientes de 30 días de nacido con caderas luxadas para mejorar el pronóstico. ${ }^{(8)}$ Sin embargo, este tratamiento no exime de complicaciones, como la osteonecrosis de cabeza femoral (NAC), la parálisis del nervio femoral, zonas de irritación cutánea entre otras. ${ }^{(9)}$

Clásicamente se ha utilizado el índice Acetabular como un indicador de displasia y sobre todo de evolución y seguimiento de la enfermedad, el objetivo de nuestro trabajo fue analizar el índice de Smith, el porcentaje de Reimers, la ceja acetabular radiográfica y la escala de Tonnis en la toma de decisión de la colocación del Arnés de Pavlik en pacientes con un Índice Acetabular elevado y con caderas estables al examen físico.

\section{Ceja Acetabular}

La ceja o sourcil, es la sombra radiográfica de tejido óseo condensado que se observa en la superficie de carga del techo acetabular en la zona superior y medial del acetábulo, mientras que el borde óseo más periférico que se evidencia en la radiografía constituye la porción anterolateral del acetábulo. El signo de la ceja en las caderas desarrolladas sin patología, se observa como una imagen radio opaca densa, semicircular, con una inclinación similar a la inclinación del borde óseo acetabular, mientras que en las caderas displásicas resalta su inclinación aumentada y su irregularidad y se la observa como una sombra radiográfica de baja densidad dada por una distribución inadecuada de cargas (Figura 1), criterio de displasia acetabular según Kim HT, et al. ${ }^{(10)}$

De forma general en las caderas displásicas se evalúan la inclinación acetabular por 2 métodos: a) clásico - relación de la esquina lateral del acetábulo, y b) método modificado - referencia la esquina de la ceja.

Ninguno de los métodos ha mostrado ser superior en virtud, que la forma de la ceja representa una pequeña fracción en relación al gran componente cartilaginoso, además que la representación de la cadera en 3 dimensiones se convierte en 2 dimensiones en la radiografía convencional y no refleja la complejidad de la deformidad. (11)
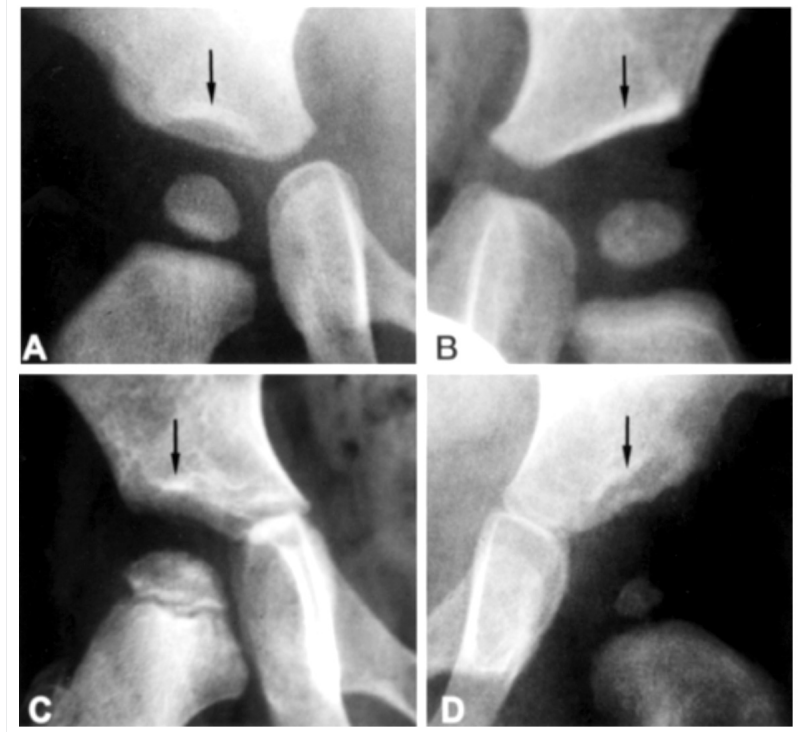

Figura 1. Ceja acetabular, A) ceja normal curvada con doble sombra, B) ceja normal recta, densidad adecuada, C) cadera displásica, falta de delineamiento claro de la ceja, sin sobreponerse al borde acetabular formando 2 puntos de referencia, D) cadera displásica con aumento de la inclinación, irregular y con 2 puntos de referencia.

Fuente:Him HT, et al. ${ }^{(10)}$

Índice Acetabular

El Índice Acetabular (IA) corresponde al ángulo formado por la línea de Hilgenreiner y una línea tangente al acetábulo, esta medición es 
frecuentemente utilizada para determinar la presencia de displasia de cadera tanto en la presentación inicial como en el seguimiento subsecuente. Un fracaso en la disminución del índice acetabular es a menudo utilizado como indicador en el cambio de la conducta terapéutica, incluso en la toma de decisión de un tratamiento quirúrgico, a pesar de esto, existen discrepancias sobre los cutoffs con mejor desempeño diagnóstico.

En 2016 Novais EN, et al. (12), estudiaron 1734 pacientes pediátricos sin patología de caderas a fin de determinar los valores normales de IA y de su correcta progresión en el tiempo obteniendo valores referenciales según edad, sexo y lateralidad. ${ }^{(12)}$ (Tabla 1)

Tabla 1 - CValores del índice acetabular normal según Novais EN, et al. ${ }^{(12)}$

\begin{tabular}{|ccccc|}
\hline $\begin{array}{c}\text { Edad } \\
\text { (meses) }\end{array}$ & $\begin{array}{c}\text { Derecha } \\
\text { (DS) }\end{array}$ & $\begin{array}{c}\text { Izquierda } \\
\text { (DS) }\end{array}$ & $\begin{array}{c}\text { Derecha } \\
\text { (DS) }\end{array}$ & $\begin{array}{c}\text { Izquierda } \\
\text { (DS) }\end{array}$ \\
$0-6$ & $24.04^{\circ} \pm 3.7^{\circ}$ & $25,64^{\circ} \pm 4^{\circ}$ & $24,14^{\circ} \pm 1.8^{\circ}$ & $23,43^{\circ} \pm 3^{\circ}$ \\
$6-12$ & $24,60^{\circ} \pm 4.2^{\circ}$ & $25,67^{\circ} \pm 3.9^{\circ}$ & $23,41^{\circ} \pm 3.7^{\circ}$ & $23,91^{\circ} \pm 4^{\circ}$ \\
DS: Desviación Estándar & & & \\
\hline
\end{tabular}

Fuente: Novais EN, et al. ${ }^{(10)}$

\section{Clasificación de Tonnis}

Permite cuantificar la severidad de la enfermedad basada en la posición de la cabeza femoral según los cuadrantes de Ombredanne (Figura 2).

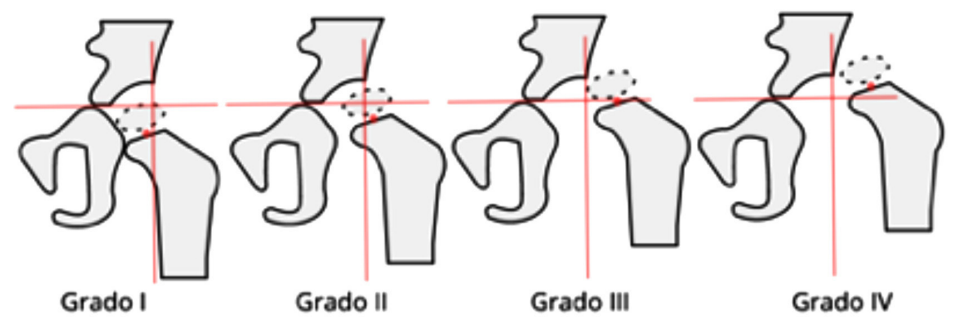

Figura 2. Clasificación de Tonnis modificada según punto de referencia en la mitad del cuello femoral.

El instituto internacional de displasia de cadera ha tomado como referencia el centro del cuello femoral en pacientes pediátricos sin osificación de la cabeza femoral la cual ha mostrado una excelente confiabilidad inter observador. ${ }^{(13)}$

-Grado I: El centro de osificación ubica medial a la línea de Perkins e inferior a la línea de Hilgenreiners (cadera normal).

-Grado II: El centro de osificación es lateral a la línea de Perkins, pero debajo de la línea de Hilgenreiners, dentro de la zona medial a $45^{\circ}$.

-Grado III: El centro de osificación es lateral a la línea de Perkins, pero debajo de la línea de Hilgenreiners, dentro de la zona lateral a $45^{\circ}$.

-Grado IV: el centro de osificación está por encima de la línea de Hilgenreiners.

\section{Porcentaje de Reimers}

Representa una fracción expresada en porcentaje (REIMERS $=$ A/B $\times 100 \%$ ) de la parte visible de la cabeza femoral respecto a la línea de Perkins (Figura 3), en donde $\mathrm{H}$ es la línea de Hilgenreiner, $\mathrm{P}$ es la línea de Perkins, y B y A son dos líneas perpendiculares a $\mathrm{H}$ tanto en su borde medial y lateral.

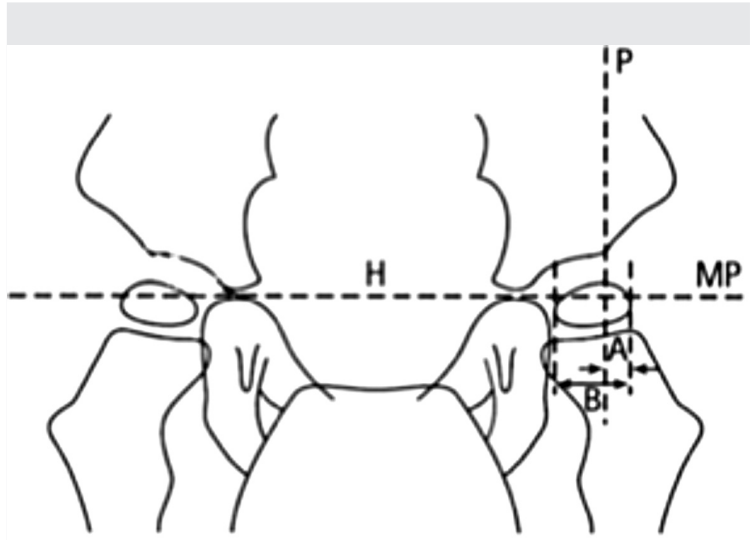

Figura 3. Porcentaje de migración de Reimers

Fuente: Autor.

Reimers en su artículo original de 1987 determinó este índice en 507 niños con radiografías de pelvis de control por problemas urológicos, pero sin antecedentes de patología en la cadera, de esta manera porcentajes que superen el $22 \%$ son considerados patológicos. ${ }^{(14)}$

Índice de Smith

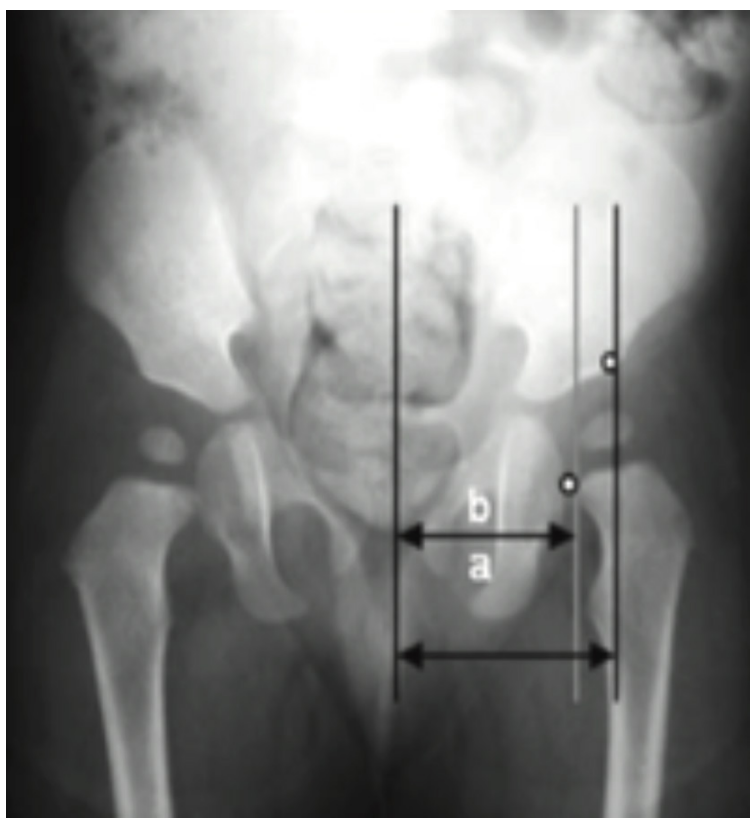

Figura 4. Índice de Smith

Fuente: Dra. Noelia Arevalo. 
Es una medida importante, ya que permite determinar si hay subluxación de cadera. Para obtenerlo hay que dibujar una línea media en la pelvis, una línea paralela a esta trazada por el borde femoral interno, y la línea de Perkins. Se mide la distancia entre la línea media y el borde femoral interno, y se designa como valor b. La distancia entre la línea de Perkins y la línea media se denomina valor a. La relación b/a debe ser menor de 0,9 (Figura 4).

Esta medida también tiene valor pronostico, estudios previos $(15,16)$ han encontrado que un índice de Smith menor de 0,9 se asocia con normalización de los parámetros radiográficos si el arco de Calvé es continuo y el índice de Smith es menor de 0,9 en los controles subsiguientes. ${ }^{(15,16)}$

Existen otras modalidades de imagen con una resolución superior a la radiografía como la resonancia magnética, no obstante, no proporciona información adicional que mejore el proceso de tomada de decisión en la práctica clínica. ${ }^{17)}$

\section{Pacientes y Métodos}

Esté fue un estudio observacional de corte retrospectiva, fueron elegibles para participar en el estudio todos los pacientes entre 3 a 12 meses de ambos sexos atendidos en la consulta externa de Traumatología y Ortopedia pediátrica del Hospital Vozandes - Quito, en el período comprendido entre 01 de enero y 31 de diciembre del 2017. Todos los pacientes fueron acompañados hasta 31 de marzo del 2018 de modo que el último paciente incluido tenga por lo mínimo 3 meses de seguimiento.

Datos clínicos, radiográficos: ángulos, porcentajes y cuadrantes se obtuvieron del prontuario electrónico de cada paciente.

Pacientes con caderas estables al examen físico, una radiografía anteroposterior de pelvis correctamente tomada y simétrica y un índice acetabular elevado según los valores propuestos por Novais EN, et al. (12), fueron incluidos en el estudio.

\section{Aspectos Bioéticos}

Este estudio sigue las directrices STROBE para estudios observacionales y fue aprobado por el Comité Revisor Institucional de Investigación del Hospital Vozandes - Quito. El consentimiento informado no fue necesario debido al diseño no intervencionista y al carácter retrospectivo del estudio.

\section{Análisis Estadístico}

Para el análisis estadístico fue utilizado IBM SPSS software package, version 23.0 for Windows (IBM, Armonk, NY, USA). Las características de los pacientes se expresaron como media y desviación estándar para variables continuas y recuentos y porcentajes para variables categóricas. La comparación entre grupos se realizó utilizando la prueba T de student o la prueba de Mann-Whitney para variables continuas. Las variables categóricas se compararon utilizando la prueba de chicuadrado de Pearson o la prueba exacta de Fisher según corresponda. Valores $p<0,05$ se consideraron estadísticamente significativos.

\section{Resultados}

Entre 01 de enero y 31 de diciembre del 2017, 104 pacientes fueron evaluados en la consulta externa de Traumatología y Ortopedia pediátrica del Hospital Vozandes - Quito.

La media de edad fue 4,63 $\pm 1,20$ meses y el $52 \%$ pertenecían al sexo masculino. Según el índice acetabular descrito por Novais EN, et al. (12), (Tabla 1): la media del índice acetabular fue $28,04^{\circ} \pm 1,73^{\circ}$, $66 \%$ y $60 \%$ de los pacientes presentaron índices acetabulares derechos e izquierdos superiores a lo normal respectivamente y $23 \%$ presentaron índices superiores en las dos caderas.

Según el tratamiento utilizado, la muestra se subdividió en: a) pacientes con dispositivos ortopédicos - arnés de Pavlik $(n=64)$ y b) sin dispositivos ortopédicos $(n=40)$.

Según el porcentaje de Reimers, la media fue de $37,9^{\circ} \pm 9,22^{\circ}$, el $87 \%$ estaba fuera de los límites de normalidad en la cadera derecha, y $91 \%$ en la cadera izquierda.

El índice de Smith se presentó normal en el 100\% de las caderas estudiadas (derecha e izquierda), la media fue de 0,79 $\pm 0,035$.

Según la clasificación de Tonnis el 90\% presentó Gl en la cadera derecha y, $85 \%$ en la cadera izquierda, GII $10 \%$ cadera derecha y $15 \%$ cadera izquierda, ningún paciente presentó GIII - GIV.

\section{Análisis Bivariable}

Al comparar pacientes con o sin arnés de Pavlik, evaluados a los 2 meses observamos que el uso de arnés no modificó los valores de índice acetabular. (OR $1.07 \mathrm{p}=0.69$ ).

Con relación al porcentaje de Reimers, ningún paciente con porcentaje normal presento un índice acetabular alterado, además la probabilidad de tener un porcentaje de Reimers alterado y de predecir una displasia residual es solo del $5 \%$ ( $p=0.53)$. 
En cuanto a la escala de Tonnis comparada con el índice acetabular a los 2 meses, se obtuvo una probabilidad de desarrollar un índice acetabular normal del 96\% para el Gl y un 60\% para el Gll, (OR $0.03 \mathrm{Gl}$, OR $0.05 \mathrm{Gll}, \mathrm{p}=0.69$ ) es decir: por cada paciente que evoluciona favorablemente con un GII en la escala de Tonnis, hay 21,3 pacientes que evolucionan favorable con una escala de Tonnis Gl. (Tabla 2).

Tabla 2 - Probabilidad de pacientes con índice acetabular alterado que usaron Arnés de Pavlik por 2 meses presentar un índice acetabular normal $\left(<25^{\circ}\right)$

\begin{tabular}{|lccc|}
\hline & OR & CHI CUADRADO & P \\
Sin arnés de Pavlik & 1,07 & 0,158 & 0,69 \\
Porcentaje de Reimers & & 0,379 & 0,538 \\
Índice de Smith & - & - & - \\
Escala de Tonnis & 21,3 & 7,28 & 0,069 \\
\hline
\end{tabular}

Fuente: Dra. Noelia Arevalo,

\section{Discusión}

La DDC engloba un amplio espectro de anormalidades en el desarrollo de la cadera, además de múltiples factores de riesgo y patologías asociadas. Existe, además discrepancia entre los puntos de cohorte para determinar cuándo una cadera es patológica y por tanto amerita algún dispositivo ortopédico o resolución quirúrgica.

Varios especialistas han intentado determinar parámetros de corte normales y patológicos sin éxito, razón por la cual, se han propuesto diferentes modalidades de imagen e índices radiográficos. ${ }^{(18)}$

A través del tiempo se han presentado una amplia variedad de resultados, por tanto, con el objetivo de unificar estos criterios, se establecieron curvas de correlación entre los diferentes índices radiográficos con el sexo, edad, y la lateralidad (12), sin embargo, los autores concluyen que no existe un parámetro seguro que permita distinguir una cadera sana de una patológica en los estadios tempranos, el presente estudio corroboró esta hipótesis al demostrar que ciertos parámetros radiográficos no son capaces de predecir el desarrollo de una cadera en pacientes con índice acetabular elevado, al mismo tiempo observamos que la unión de varios parámetros: ceja acetabular, escala de Tonnis y el índice acetabular discretamente elevado podría ser una variante de la normalidad en algunos infantes, sin embargo, no podemos recomendar el desuso de dispositivos ortopédicos dado la amplia evidencia existente en la literatura médica que respalda la mejoría en el desarrollo de las caderas.

El presente estudio reflejó un leve predominio en el número de caderas displásicas en el sexo masculino, hallazgo discordante con la mayoría de estudios previos, donde el predominio es en el género femenino, probablemente a que la clasificación original propuesta por Novais EN, et al. ${ }^{(12)}$, se la realizó con rangos de referencia menores para el género masculino, lo cual aumentó la sensibilidad en la detección de displasia en este grupo.

A pesar de la evidencia publicada, todavía se sabe poco de la historia natural de esta patología, sobre todo en pacientes con índices acetabulares limítrofes y con caderas estables clínicamente, por este motivo el tratamiento es controverso en muchos casos.

Estudio previo (15) demostró que este tipo de pacientes evolucionan favorablemente a mediano plazo en la evolución natural de la enfermedad sin tratamiento ortopédico, poniendo en tela de duda de la fiabilidad del índice acetabular (15), Wilmington trato de responder a esta pregunta al evaluar la inestabilidad mediante el método Harcke positivo (inestabilidad con estrés, pero se reduce en reposo) en pacientes con índice acetabular elevado $o$ en valores limítrofes. Los autores concluyeron que a los 36 meses se obtuvo una normalización del índice acetabular en el $100 \%$ de las caderas estudiadas (19), mientras que el presente estudio demuestra datos más alentadores a corto plazo con 95,3\% de probabilidad de desarrollar una cadera normal, con un índice acetabular menor de $25^{\circ}$, luego de no haber recibido ningún tratamiento, de esta manera se ratifica que existen otras variables que puede jugar un rol más importante al momento de iniciar un tratamiento ortopédico.

La displasia acetabular residual ha sido el centro de atención en estos pacientes, debido a que el índice acetabular es un indicador de referencia en el tratamiento tanto quirúrgico como ortopédico, y se desconoce puntos de corte específicos o parámetros asociados para la elección de un tratamiento $u$ otro a pesar que $25^{\circ}$ ha sido una referencia estándar de displasia residual. ${ }^{(20)}$

Pese a los grandes avances tecnológicos, la radiografía continua siendo el estudio de elección para el acompañamiento de estos pacientes, hasta el momento no ha podido ser remplazada aun por la resonancia ni la ecografía $(5,21)$, sin embargo, estudios en pacientes con caderas normales limítrofes donde el tratamiento quirúrgico es controversial, la resonancia magnética ha demostrado de manera objetiva el desarrollo normal o anormal de los diferentes centros de osificación de la cadera y de esta manera se ha podido mejorar el proceso de tomada de decisión. De igual forma se ha podido predecir de manera adecuada la evolución del acetábulo. (22)

Estos avances se han dado gracias a la comprensión adecuada del limbus y del neolimbus y de su implicación tanto en la valoración por imagen como del manejo de este durante las diversas intervenciones quirúrgica, permitiendo obtener mejores resultados al respetar estas estructuras. ${ }^{(23)}$

En el presente estudio, ante la sospecha de una cadera displásica, la ceja acetabular, y la escala 
de Tonnis pueden auxiliar en el diagnóstico de pacientes que presentan índices levemente elevados, por otro lado, estos pacientes podrían beneficiarse de estudios más específicos como la resonancia magnética, la cual nos brindaría la oportunidad de correlacionar objetivamente los resultados durante el seguimiento y de esta manera reducir las incertezas en la indicación de tratamientos ortopédicos.

\section{Conclusión}

La inestabilidad y la displasia residual de cadera requiere tratamiento y seguimiento cercano de su evolución, el examen físico tiene una sensibilidad de apenas de 60\%, la ecografía ha dado una amplia ventaja en el estudio de estos pacientes, más aún con pruebas dinámicas, ya que puede responder a 3 preguntas claves cuando el diagnóstico es incierto y estas son: a) el acetábulo es normal, b) la cadera es estable, luxable o subluxable, y c) si esta luxada, determinar si es reductible. La desventaja de la ecografía es el ser un procedimiento operador dependiente24, aunque sabemos que la displasia residual y la inestabilidad de cadera representa un riesgo de 2,5 veces mayor de presentar coxartrosis severa, y artroplastia de cadera a largo plazo de acuerdo con los resultados de Engesaeter $\mid \varnothing$, et al. ${ }^{(25)}$, este estudio muestra resultados discordantes con la literatura. Observamos que el tratamiento ortopédico puede ser diferido en caderas con parámetros específicos, debido a su evolución natural cuando comparadas con el grupo control (Arnés de Pavlik), por otro lado, se eliminarían también las complicaciones propias del tratamiento ortopédico, como la necrosis de la cabeza femoral y la parálisis del nervio femoral.

\section{Referencias}

1. Herring JA, Tachdjian's M. Pediatric Orthopaedics. 5th Edition. Sevier E, editor. Dallas, Texas; $2014.662 \mathrm{p}$.

2. Loder RT, Skopelja EN. The epidemiology and demographics of hip dysplasia.
ISRN Orthop. 2011 Oct 10;2011:238607. doi:10.5402/2011/238607.

3. Andrango S, Ordoñez F. Determinación de la prevalencia de displasia de cadera en desarrollo en niños de 3 a 6 meses mediante estudio clínico y radiológico para diagnostico precoz y prevención de complicaciones en la consulta externa de pediatría del Hospital Baca Ortiz Quito. Universidad Central de Ecuador; 2014.

4. Vaquero-Picado A, González-Morán G, Garay EG, Moraleda L. Developmental dysplasia of the hip: Update of management. EFORT Open Rev. 2019;4(9):548-56.

5. Upasani VV, Bomar JD. Reliability of plain radiographic parameters for developmental dysplasia of the hip in children. J Child Orthop. 2012;173-6.

6. Ömeroglu H. Treatment of developmental dysplasia of the hip with the Pavlik harness in children under six months of age: indications, results and failures. J Child Orthop. 2018;12(4):308-16

7. Ran L, Chen H, Pan $Y$, Lin $Q$, Canavese $F$ Chen S. Comparison between the Pavlik harness and the Tübingen hip flexion splint for the early treatment of developmental dysplasia of the hip. J Pediatr Orthop B. 2019;1.

8. Larson JE, Patel AR, Weatherford B, Janick JA. Timing of Pavlik Harness Initiation: Can We Wait? J Pediatr Orthop. 2019;39(7):335-8.
- Tiruveedhula M, Reading IC, Clarke NMP. Failed pavlik harness treatment for DDH as a risk factor for avascular necrosis. J Pediat Orthop. 2015;35(2):140-3.

10. Kim HT, Kim J II, Yoo C II. Diagnosing childhood acetabular dysplasia using the lateral margin of the sourcil. J Pediatr Orthop. 2000;20(6):709-17.

11. Kim SM, Sim EG, Lim SG, Park ES. Reliability of hip migration index in children with cerebra palsy: The classic and modifi ed methods. Ann Rehabil Med. 2012;36(1):33-8.

12. Novais EN, Autruong PT, Pan Z, Chang FM Meyers ML. Normal Percentile Reference Cur ves and Correlation of Acetabular Index and Acetabular Depth Ratio in Children. J Pediatr Orthop. 2016;38(3):163-9.

13. Narayanan U, Mulpuri K, Sankar WN, Clarke NMP, Hosalkar H, Price CT. Reliability of a new radiographic classification for developmenradiographic classification for developmen-
tal dysplasia of the hip. J Pediatr Orthop. tal dysplasia of the
2014;35(5):478-84.

14. Eklöf $O$, Ringertz H, Samuelsson L. The percentage of migration as indicator of femora head position. Acta radiol. 1988;29(3):363-6.

15. Mladenov K, Dora C, Wicart P, Seringe R. Natural history of hips with borderline acetabular index and acetabular dysplasia in infants. Pediatr Orthop. 2002;22(5):607-12.

16. Boniforti FG, Fujii G, Angliss RD, Benson MKD. The reliability of measurements of pelvic radiographs in infants. J Bone Jt Surg. vic radiographs
$1997: 79(4): 570-5$.

17. Kotlarsky P, Haber R, Bialik V, Eidelman M, Kotlarsky $P$, Haber $R$, et al. Developmental dys- plasia of the hip: What has changed in the last 20 years? 2015;6(11):886-901.

18. Rae Duncan. The acetabular index critical review. J bone jt surg. 1959

19. Blazej Pruszczynski, H Theodore Harcke, Lau rens Holmes, Natural history of hip instability in infants (without subluxation or dislocation): a three year follow-up. BMC Musculoskelet Disord. 2014;15: 335.

20. Li Y, Guo Y, Li M, Zhou Q, Liu Y, Chen W. Acetabular index is the best predictor of late residual acetabular dysplasia after closed reduction in developmental dysplasia of the reduction in developm
hip. Int Orthop. 2017;10.

21. Pirpiris $M$, Payman KR, Otsuka NY,. The Assessment of Acetabular Index: is there still a place for plain radiography?. J pediatr Orthop 2006;26(3):310-5.

22. Douira-Khomsi W, Smida M, Louati H, Hassine L Ben, Bouchoucha S, Saied W, et al. Magnetic resonance evaluation of acetabular residua dysplasia in developmental dysplasia of the Hip: A preliminary study of 27 patients. J Pediatr Orthop. 2010;30(1):37-43.

23. Landa J Benke M. Feldman DS. The limbus and the neolimbus in developmenta dysplasia of the hip. Clin Orthop Relat Res. 2008;466(4):776-81.

24. Paton RW. Management of neonatal hip instability and dysplasia. Early Hum Dev. 2005;81 (10):807-13

25. Engesaeter $1 \varnothing$, Lie SA, Lehmann TG, Furnes $O$ Vollset SE, Engesaeter LB. Neonatal hip instability and risk of total hip replacement in young adulthood. Acta Orthop. 2008;79(3):321-6. 